\title{
A Case of Open Angle Glaucoma Secondary to Posner-Schlossman Syndrome
}

Andrew J. Rixon, OD, FAAO

Memphis VA Medical Centre

Rebecca A. Wenig, OD

Coley \& Coley Family Eye Care

Brittany N. Rigdon, OD

Precision Eye Care Inc.

\section{ABSTRACT}

This is a case report demonstrating the appropriate diagnosis and management of PSS and its potential sequelae. Posner-Schlossman Syndrome is characterized by recurrent, unilateral attacks of significantly increased intraocular pressure. The etiology and pathophysiology of this condition remains unclear. Duration of this chronic condition and the risk of developing secondary open angle glaucoma are directly proportional. The patient and managing optometrist must be aware of the necessity of timely intervention during acute attacks, as well as adherent longitudinal care to preserve function and quality of life. The ensuing case report and review underscores the impact of this disease.

\section{KEYWORDS:}

posner schlossman syndrome (pss), open angle glaucoma (oag), cytomegalovirus (cmv), intraocular pressure (iop), trabeculitis 


\section{INTRODUCTION}

Posner-Schlossman Syndrome (PSS) was first described as a glaucomatocyclitic crisis by Terrien and Veil in 1929 and was later re-named for Adolph Posner and Abraham Schlossman after their case series of nine patients with the condition. ${ }^{1,2}$ PSS typically affects young to middle-aged individuals between the ages of 20 and 50, although there are reports involving adolescents and patients older than $70 .{ }^{3-5}$ PSS is diagnosed by its clinical presentation, which is characterized by an idiopathic, recurrent, unilateral and self-limiting attack of increased intraocular pressure (IOP) accompanied by mild non-granulomatous anterior uveitis. PSS may include mild patient symptoms such as visual blur and eye pain. ${ }^{1,3}$ Slit lamp evaluation may detect minimal keratic precipitates with rare cell and flare in the anterior chamber; posterior synechiae is unlikely. ${ }^{3}$ Gonioscopic evaluation reveals an open angle without peripheral anterior synechiae formation, and keratic precipitates may also be seen. ${ }^{3}$

Patients with PSS characteristically have IOPs surpassing 40mmHg during their attacks. Readings will often be much higher, although slightly lower IOPs have also been reported. ${ }^{6}$ The literature shows mean IOP ranging between 42.77 and $49.20 \mathrm{mmHg} .{ }^{6-8}$ Between the recurrent PSS attacks, which tend to resolve within days to weeks, glaucoma-associated parameters remain within normal limits. ${ }^{9}$ However, as many as $45 \%$ of PSS patients have been shown to have associated open angle glaucoma (OAG). ${ }^{6}$ The development of OAG caused by repeated attacks of PSS has been noted throughout the literature. ${ }^{3,6,10}$

In a retrospective non-comparative case series on the clinical course of 53 PSS patients with an average duration of seven years, $26.4 \%$ were diagnosed as having developed OAG secondary to PSS. ${ }^{3}$ The literature reports that patients who have had PSS for more than 10 years have a 2.8 -fold greater risk of developing glaucoma than their counterparts who have had the disease less than 10 years. ${ }^{6}$ The following case demonstrates the appropriate diagnosis and management of PSS and its possible sequelae.

\section{CASE REPORT}

A 49-year-old African-American male was originally diagnosed with PSS OS by an outside provider in 1997, and with OAG secondary to recurrent cyclitic attacks OS by our clinic in 2013. The patient had a documented history of poor compliance with longitudinal care and inconsistently presented during PSS attacks for management. This patient's highest recorded intraocular pressures (IOP) were $18 \mathrm{mmHg}$ OD and $38 \mathrm{mmHg}$ OS by Goldmann applanation tonometry (GAT or TAG) during his most recent cyclitic attack in February 2016. He was treated with loteprednol $0.5 \%$ one drop eight times per day and continued timolol twice per day OS in response to that attack. He returned in a week with a normalized IOP of $10 \mathrm{mmHg}$ in the OS and the resolution of ocular signs consistent with his attack.

The patient presented in August 2016 for a glaucoma follow-up and reported adherent use of 0.5\% timolol maleate twice daily in his left eye. The patient denied flashes, floaters, haloes, headaches, double vision, burning, itching, redness and tearing in either eye. He believed that his PSS attacks coincided with the control of his systemic conditions.

His medical history was pertinent for chronic shoulder pain (post rotator cuff surgery) and knee pain due to osteoarthritis, migraines and depressive disorder. A diagnosis of seronegative $(<8.6 \mathrm{IU} / \mathrm{mL})$ rheumatoid arthritis (RA) was made in 2001. However, there was a debate amongst his rheumatologists whether his diagnosis was seronegative RA or mixed connective tissue disease (MCTD), as the patient is (+)ANA (antinuclear antibody test) and (+) RNP (ribonucleoprotein). The patient is currently followed by rheumatology every three months. There is no relevant family ocular or systemic history.

The examination in August 2016 revealed 20/20 visual acuity OD and OS. There were no defects involving the pupils, extraocular muscles or confrontation fields. Slit lamp examination was remarkable only for trace fine keratic precipitates and 0.5 anterior chamber reaction OS (AC grade per Standardization of Uveitis Nomenclature (SUN) working group), with the irises intact and of equal color OU. IOP in office measured $17 \mathrm{mmHg}$ OD and $10 \mathrm{mmHg}$ OS @1:07pm. Dilated fundus exam (DFE) showed a right optic nerve head measuring 1.50mm vertically (with a 66D funduscopic lens) with a $0.55 \mathrm{~V} / 0.55 \mathrm{H} \mathrm{C} / \mathrm{D}$. The lamina exhibited no major reconfiguration, the neuroretinal rim was intact 360 and RNFL was diffuse and symmetrical in both the superior and inferior hemispheres. The left optic nerve head measured $1.50 \mathrm{~mm}$ vertically using a $66 \mathrm{D}$ lens with a $0.85 \mathrm{~V} / 0.80 \mathrm{H} \mathrm{C} / \mathrm{D}$. There was concentric erosion of the rim with posterior laminar excavation and striate reconfiguration. The residual rim appeared perfused and the RNFL grossly exhibited diffuse inferior thinning in excess of that found in its superior counterpart. (Figures 1 and 2) The periphery was flat, intact and had no holes, tears, or detachments OU. 
Additional testing determined the patient's central corneal thickness to be $544 / 557 \mu \mathrm{m}$ OD, OS. Corneal hysteresis and corneal compensated IOP (IOPcc) were 8.2 and $18.3 \mathrm{mmHg}$, respectively, OD, and 8.6 and $11.4 \mathrm{mmHg}$, respectively, OS.

The most recent gonioscopy, using the Shaffer classification, was grade 4 with a wide open angle to ciliary body with 1+ trabecular meshwork pigmentation 360' OD and a grade 3 open angle to scleral spur with 1+ pigmentation 360' with no synechiae OS. Trabecular meshwork was notably clear in both eyes. Visual field testing was done and reliable in both eyes (Figure 3). The right eye had a mean deviation of $+0.37 \mathrm{~dB}$ and no glaucomatous defects. The left eye had a mean deviation of $-2.66 \mathrm{~dB}$ with 3 local defects within the nasal step region of $<1 \%$ probability on pattern deviation. This was consistent with past findings. Optical coherence tomography (Spectralis ${ }^{*}$, Heidelberg Engineering GmbH, Heidelberg, Germany), specifically circumpapillary RNFL (cp-RNFL), of the OD had a global thickness of $92 \mathrm{um}$, which was unchanged from a previous scan one year earlier. The OS scan had a global cp-RNFL thickness of $55 \mathrm{um}$, which was also unchanged from April 2015 (Figure 4). A posterior pole asymmetry analysis (PPAA) scan showed severe thinning of the total retinal thickness OS compared to OD (Figure 5). The average total retinal thickness by PPAA was 287 um OD and 267 um OS.

Figure 1: Posterior pole photos showing OS RNFL asymmetry superior > inferior.
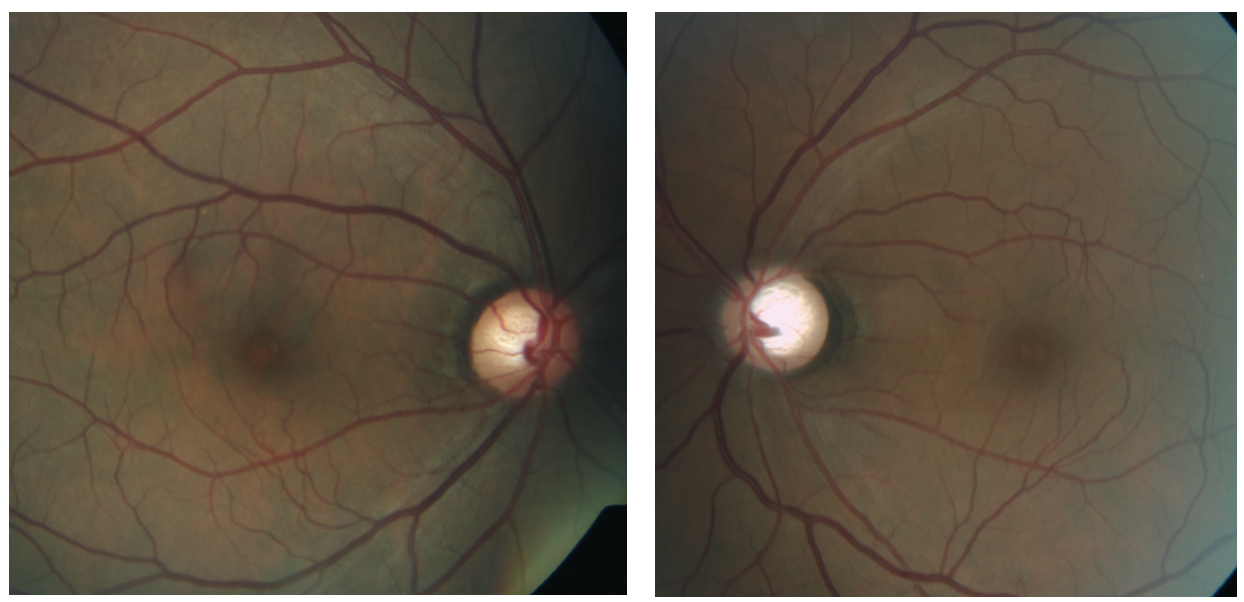

Figure 2: 20' ONH photos. OS exhibits concentric neuro-retinal rim loss and substantial laminar reconfiguration.
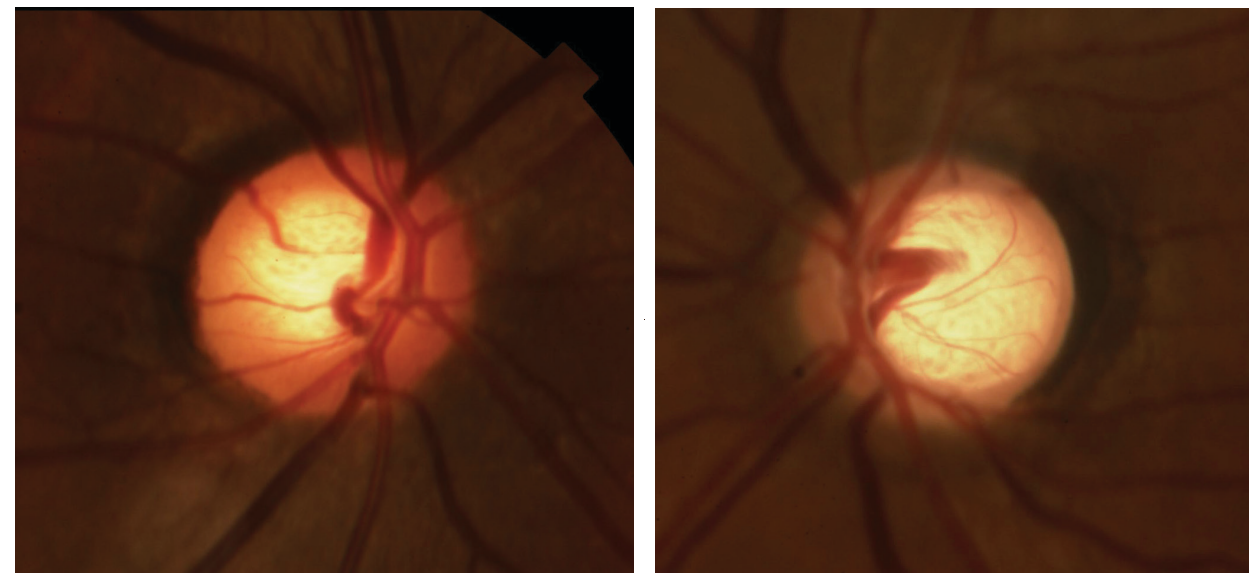


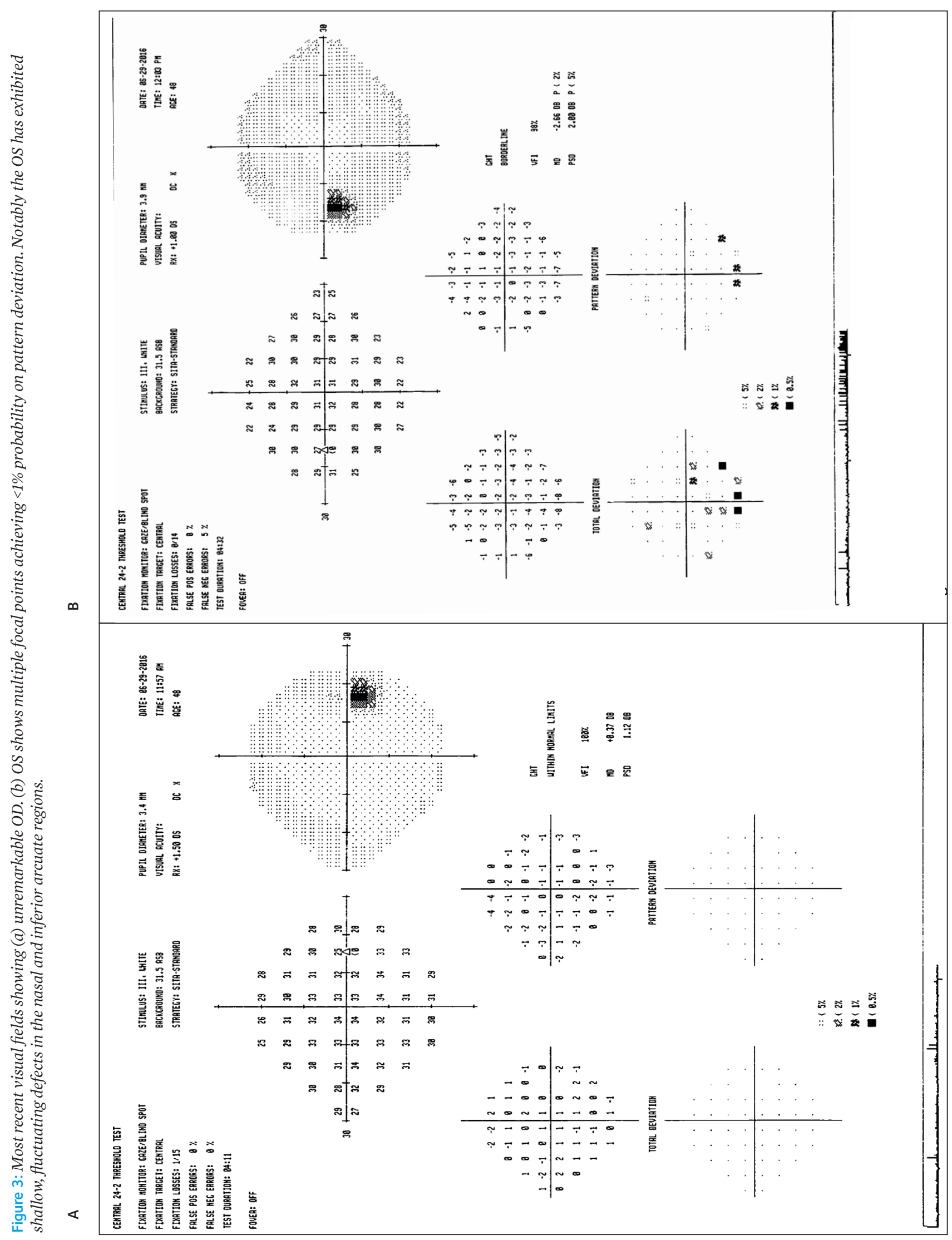


Figure 4: Circumpapillary RNFL (cp-RNFL) analysis OD and OS. Note significant thinning globally as well as in the ST and IT sectors $O S$.

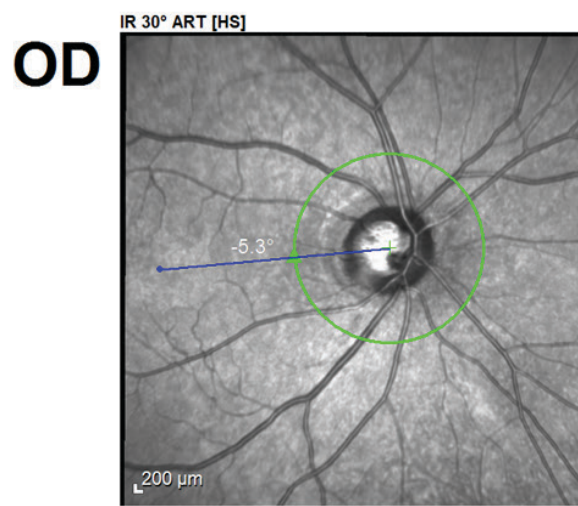

OCT ART (100) Q: 30 [HS]
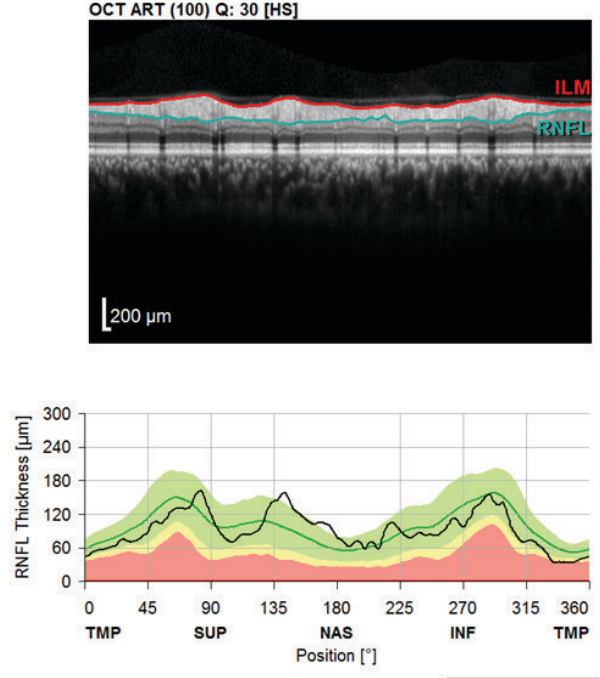
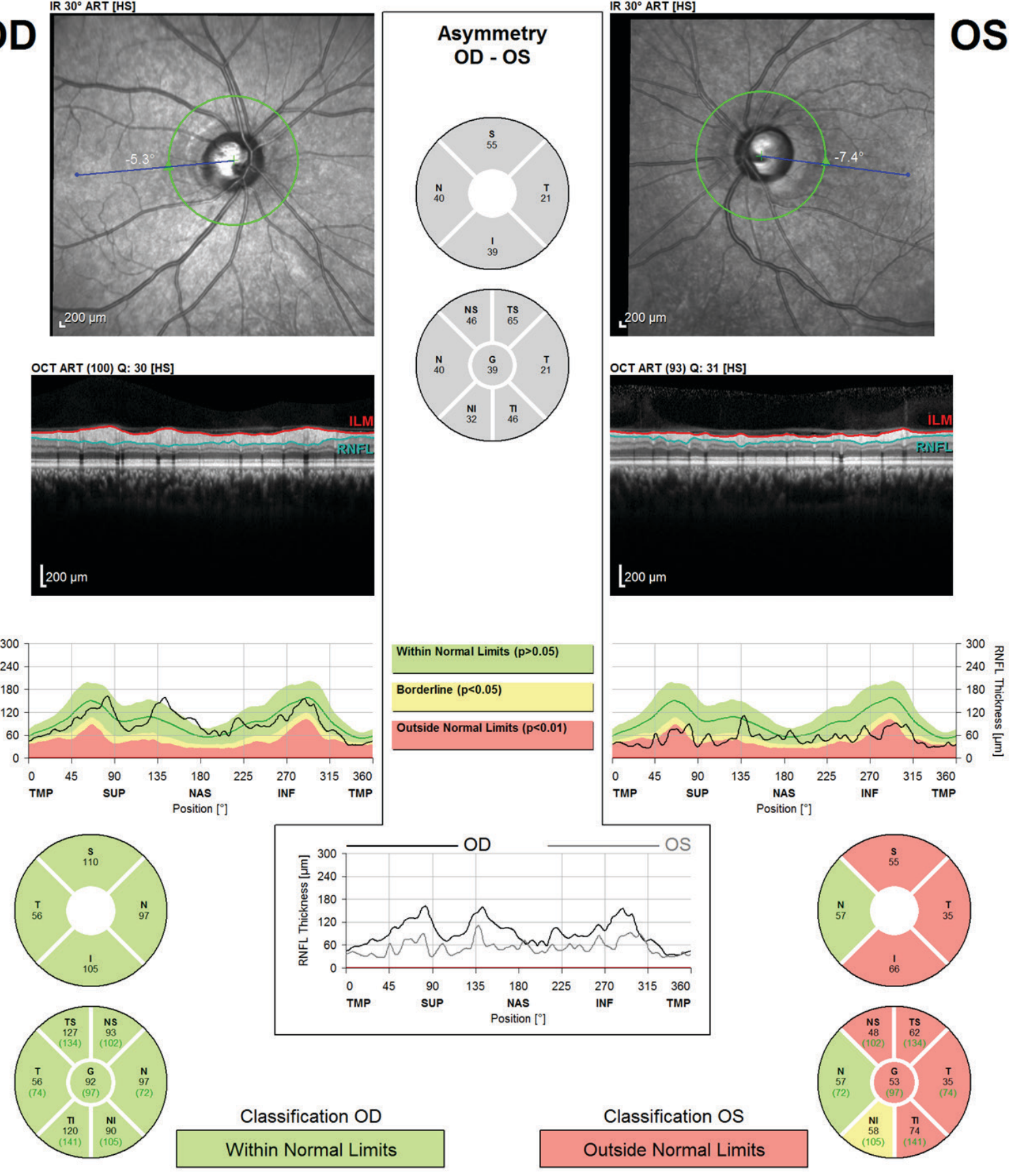
Figure 5: Posterior pole asymmetry analysis shows severe depression in both the superior and inferior arcuate regions OS.
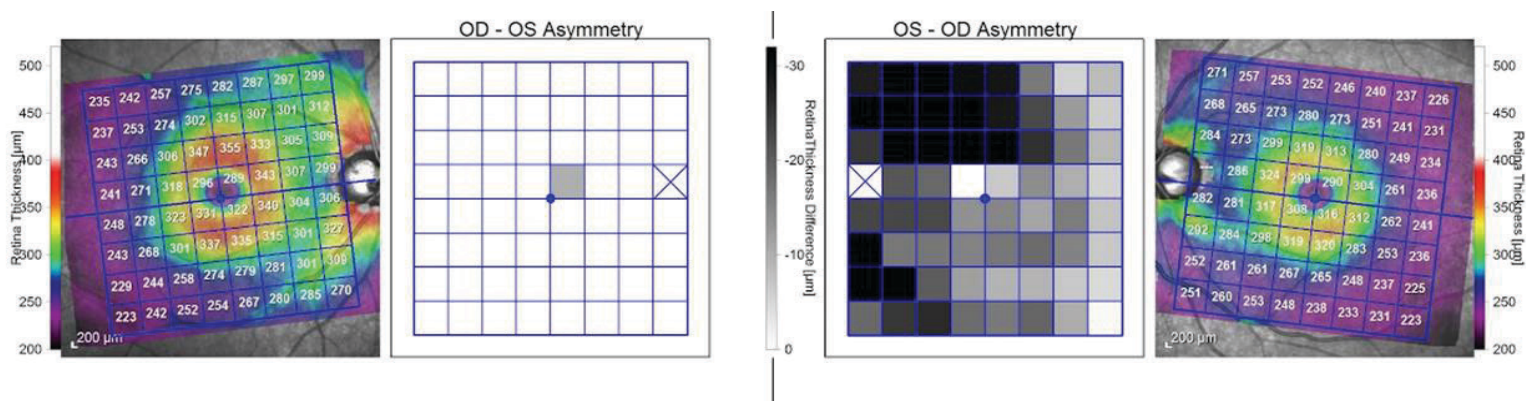

At the conclusion of the exam, the patient's PSS and SOAG were considered to be stable. The patient was instructed to continue timolol $0.5 \%$ b.i.d. OS and scheduled to return in three months for a glaucoma progress visit. The patient was educated to seek care immediately if a new attack occurred. Lastly, he was educated that the relationship between his RA/MCTD and PSS has not yet been described in the literature, and any connection between the two was dubious.

\section{DISCUSSION}

The exact mechanism of increased IOP in PSS is unknown. ${ }^{9}$ It is understood that, in cases of inflammatory open angle glaucoma, trabecular meshwork (TM) obstruction is the most common mechanism of increased IOP. TM obstruction is followed by hypersecretion of aqueous and mediated by prostaglandins $\mathrm{E}_{1}$ and $\mathrm{E}_{2}$ (PGE1 and 2). ${ }^{11}$ Specifically, the blood-aqueous barrier is disrupted, which permits an influx of inflammatory cells into the aqueous and leads to the harboring of normal serum components in the outflow system. Trabeculitis likely develops, resulting in pore-narrowing and dysfunction, further obstructing aqueous outflow. ${ }^{9}$ Significantly elevated aqueous prostaglandin levels have been documented during the acute phase of PSS. This increased PGE concentration causes a concomitant rise in the aqueous protein concentration and subsequent increased aqueous viscosity. ${ }^{11}$ Increased aqueous production coupled with decreased aqueous outflow results in a substantial increase in IOP. ${ }^{12}$

The precise etiology and pathophysiology of PSS are also still unclear. ${ }^{3,13}$ Several potential causes have been proposed, with more recent theories focusing on viral etiologies for PSS. Additional mechanisms that have been explored include an abnormal vascular response, autosomal defects, and oxidative damage of the trabecular meshwork. ${ }^{3,13}$

Research on possible viral etiologies for PSS has recently focused on cytomegalovirus (CMV). This focus began because studies using PCR have shown that up to $75 \%$ of aqueous samples from immunocompetent PSS patients were positive for CMV viral DNA during active attacks. ${ }^{7,13-16}$ Interestingly, further PCR exploration of aqueous samples from PSS patients during inactive periods found that all patients were negative for CMV viral DNA. ${ }^{16}$ These results are compelling and possibly suggestive of an association between intraocular CMV infection and PSS in healthy patients. However, the virtually universal sero-prevalence of human CMV precludes a conclusion of causation at this time. ${ }^{7,8,17}$ Although real-time quantitative detection of CMV by plasma PCR has increased the sensitivity and specificity of testing, there is no consensus that would support routinely ordering this bloodwork in patients with PSS. ${ }^{18}$

Despite evidence associating CMV infection with PSS, the ultimate clinical inflammatory reaction appears to be immune-mediated and not infectious in nature. ${ }^{18}$ While trabeculitis is thought to occur, the precise pathophysiological mechanism for this response is unknown. In a study of 53 immunocompetent uveitis patients with clinical signs of PSS, aqueous samples were compared to those of 25 healthy control patients for the presence or absence of CMV viral DNA, as well as cytokine concentrations. Over 97\% of PSS samples were distinguished from controls by elevated cytokine levels, not CMV. The authors found that the presence or absence of CMV was not significantly associated with different aqueous cytokine concentrations in these patients. ${ }^{18}$ This pro-immune inflammatory conclusion is consistent with PSS's classic hyper-response to corticosteroid 
treatment, which helps differentiate PSS from other forms of CMV uveitis that fail to respond to steroids..$^{19}$ In our patient, both of his proposed systemic conditions (RA and MCTD) are, not surprisingly, associated with an increased production of a range of cytokines. ${ }^{20,21}$ However, PSS has not been studied in cohorts similar to our patient.

Vascular endothelial dysfunction is a known risk factor for both primary open angle glaucoma and normal tension glaucoma. It has also been proposed to be involved in PSS. ${ }^{8,22} \mathrm{~A}$ small, prospective case-control study in 2010 involving 12 patients with PSS and 15 controls was undertaken to evaluate brachial artery endothelium-dependent flowmediated dilation (FMD) as an indicator of vascular endothelial function. ${ }^{22}$ The results showed that FMD in the PSS group was much lower than that in the control group. The authors concluded that these results imply peripheral vascular endothelial dysfunction in patients with PSS. ${ }^{22}$ It has been proposed that this endothelial dysfunction could predispose PSS patients to developing glaucomatous optic neuropathy secondary to their IOP spikes. ${ }^{8}$ Further studies will be needed to advance these speculations.

The role that genetic factors play in the etiology of PSS has also been explored. In 2015, a study on the association of human leukocyte antigens (HLA) allelic diversity revealed that the frequency of HLA-C*1402 in PSS patients was significantly higher than that in controls. ${ }^{23}$ Although the cohort was comprised of a southern Chinese population and the results have not been validated outside of this population, these findings suggest that genetic immune regulation may play a role in these patients. The rate of PSS in Japan and Singapore ranges from 1.7-4.3\% of all uveitis diagnoses, which is similar to the findings in northern Europe. ${ }^{8}$ Although this point has not been extensively researched, it seems reasonable that, based on similar global rates of the same condition, a genetic component may be universal.

The exclusion of differential diagnoses of hypertensive uveitis is the key to successful patient management. Other viral etiologies for uveitis, including herpes simplex and herpes zoster, can present similarly to PSS. In these cases, as in PSS, elevated IOP may result from normal aqueous production combined with impaired outflow due to an inflamed trabecular meshwork. ${ }^{24}$ The incidence of elevated IOP in herpetic keratouveitis cases has been reported to be up to $43 \% .{ }^{25}$ However, unlike with PSS, these entities will typically present with more pronounced symptoms of pain and photophobia and additional anterior segment signs. Herpes simplex uveitis presents with corneal dendrites, iris atrophy, and large keratic precipitates. ${ }^{26}$ Herpes zoster virus (HZV)-associated uveitis will present with corneal pseudodendrites. Most often, these patients have a prior instance of a dermatological zoster condition. ${ }^{26}$ Further confirmation of HZV can be made by testing corneal sensation, which will be markedly decreased. Fuchs heterochromic iridocyclitis (FHI) is another important and, arguably, most confounding differential diagnosis. FHI, like PSS, presents unilaterally with signs of mild inflammation and mild patient symptoms. ${ }^{27}$ In fact, iris heterochromia can develop in PSS, further complicating diagnosis and management. ${ }^{1,27}$ However, unlike PSS, FHI tends to be chronic and refractory to steroids, manifests lower IOP and often develops posterior subcapsular cataract.,27 Finally, although this is not common, acute angle closure should be considered. In contrast to PSS, acute angle closure presents with a sudden increase in intraocular pressure, shallow anterior chamber, and narrowed-to-closed anterior chamber angles. ${ }^{3}$ Nausea, corneal edema and severe ocular pain are symptoms that are most commonly associated with an acute angle closure event-all of which are inconsistent with PSS.,32

Following an accurate diagnosis of PSS, treatment is aimed at managing both the inflammation and IOP elevation. ${ }^{3,8}$ Apraclonidine hydrochloride $1 \%$ has been universally shown to cause a rapid decrease in IOP within an hour of instillation, leading multiple authors to instill it during acute PSS attacks. ${ }^{29,30}$ Additional IOP-lowering medications, such as beta-blockers and carbonic anhydrase inhibitors, are also commonly used to treat acute attacks. ${ }^{3}$ Topical corticosteroids are dosed at a minimum of 4-6 times per day, as recommended in the literature, followed by a rapid taper. ${ }^{3,31}$ Rapid taper is justifiable due to the typical self-limiting short course of PSS attacks ranging from days to weeks. ${ }^{3}$ The managing physician must additionally be aware that corticosteroid use administered to mitigate inflammation during acute attacks can actually result in a paradoxical decrease in the facility of aqueous outflow, which raises IOP. ${ }^{11}$ No previous study has addressed the superiority or inferiority of one topical corticosteroid versus another in the specific management of PSS. The self-limiting nature of PSS attacks helps justify our use of loteprednol, which is known to have a substantially lower likelihood of inducing clinically meaningful IOP elevation..$^{32}$ All of the known topical steroids have the potential to cause steroid-induced glaucoma. ${ }^{33}$ 
Timely reduction of IOP during attacks with the above agents is imperative as negative optic disc topography and flowmetry changes that result from substantially elevated IOP are reversible. ${ }^{34,35}$ There is no compelling evidence to prescribe chronic low-dose topical steroids in patients with PSS, since they have not been shown to reduce the frequency or severity of attacks. Additionally, the chronic use of IOP-lowering agents has not been shown to be effective at preventing PSS recurrence and therefore is not necessary in the absence of coexisting POAG or resulting SOAG. ${ }^{3}$ Furthermore, there are no guidelines for setting a target pressure in PSS patients who develop glaucoma. We subscribe to Jampel's conceptual description of target IOP: “That IOP at which the quality of life (QOL) from preserved vision and the QOL from not having side effects from treatment is maximized". ${ }^{36}$

The use of antiviral medications in the management of PSS has been investigated following more recent research focusing on CMV as a primary etiology for the disease. The elimination of CMV from the anterior chamber using valgancyclovir can modify the disease by reducing IOP, the number of medications used, controlling inflammation, and lowering the recurrence rate ${ }^{8,37-39}$ A retrospective study comparing topical, oral, and intravitreal valgancyclovir in patients with uveitis secondary to CMV showed a higher failure rate (36\% topical v. $9 \%$ oral) but decreased rate of recurrence in the topical group over 27.7 months of followup. ${ }^{39}$ The study used $0.15 \%$ ganciclovir gel, commercially available internationally as Virgan (known as Zirgan in the United States). The recurrence of uveitis once medications were discontinued approached $75 \%$, likely as a result of valganciclovir being virostatic rather than virocidal. ${ }^{8,39}$ A study on the long-term use (3 months) of compounded topical $2 \%$ ganciclovir in 68 eyes of CMV+ PSS patients found that treatment was effective for clearing the viral load, helping to control IOP, and preserving the corneal endothelium. ${ }^{40}$ The authors proposed that early implementation of ganciclovir could lower the risk of advanced glaucoma and limit the need for surgery in chronically recurrent cases. ${ }^{40}$

A retrospective observational case study from 2014 was conducted on 11 patients with clinically diagnosed PSS who were previously refractory to acyclovir. These patients were subsequently treated with $900 \mathrm{mg}$ of oral valganciclovir twice daily for three weeks, followed by $450 \mathrm{mg}$ twice daily for a mean of 20 months. Notably, 6 of the 11 patients underwent anterior chamber taps and 5 of these 6 were positive for CMV on PCR analysis. In 7 of the 11 patients, valganciclovir led to resolution of inflammatory activity and stable IOP. This permitted tapering and discontinuation of valganciclovir in 6 of the 7. Although two patients had inflammatory recurrences and needed retreatment, only one remained on reduced therapy. Ultimately, no recurrences were observed during the 10-month post-treatment observation period in the patients who responded to valganciclovir. The authors concluded that a course of at least 12 months of oral valganciclovir might reduce the recurrence rate in patients who continue to relapse on topical ganciclovir or short-term oral valganciclovir. ${ }^{38}$

Given the results of these multiple case studies, it has been suggested that there should be a low threshold for anterior chamber tap in patients whose PSS attacks are not improving and suggestive of CMV infection. ${ }^{8}$ Furthermore, the concurrent empirical use of topical ganciclovir in these cases has been suggested, followed by a minimum 3 -month course of oral medications if there is a poor response to topical medications. ${ }^{8}$ Although currently accepted methods of management are still primary, the ability to have positive outcomes with anti-CMV agents may represent an important future option in the management of PSS patients with recurrent disease. Unfortunately, there are no clinically detectable differences between CMV+ and CMV- patients in PSS, so it is still difficult to identify which patients will benefit from more invasive testing. ${ }^{41}$

Surgical intervention may be indicated in PSS cases that show increased attack frequency, when IOP fluctuations during attacks are significant, or in cases where there is progressive glaucomatous damage to the optic nerve and/or visual field due to uncontrolled IOP. ${ }^{3}$ Filtration surgery has been shown to be successful in PSS cases. A 2001 study involving 9 patients with PSS undergoing trabeculectomy with mitomycin C (MMC) showed an $80 \%$ success rate in reducing the frequency of PSS events as well as preventing IOP spikes during the cyclitic attacks. ${ }^{6}$ Consistent with PSS, the patients undergoing filtration in the above study required no anti-glaucoma or anti-inflammatory treatment between episodes. ${ }^{6} \mathrm{~A}$ possible explanation for why filtration is successful in these cases is that inflammatory cells migrate through the postoperative bleb, thus reducing anterior chamber activity and trabeculitis, and possibly protect the bleb from failure. ${ }^{6,8}$ However, surgical therapy should involve careful consideration as outcomes may not improve and the condition may even worsen postoperatively. In uveitic glaucoma patients, conjunctival cellular changes such as increased fibroblast, lymphocyte, and macrophage counts have been implicated in surgical failure in patients who have undergone filtration. ${ }^{2}$ Filtration surgery is associ- 
ated with postoperative complications such as wound leak, flat anterior chamber, hypotony, choroidal effusion, hemorrhage, hyphema and infection. ${ }^{42}$

Recently, trabeculectomy ab interno (trabectome) has been assessed as another, less invasive, surgical option for patients on oral valgancyclovir with uncontrolled CMV+ PSS. Seven patients underwent trabectome surgery and were followed for 12 months postoperatively. The mean IOP before surgery was $40 \pm 10 \mathrm{mmHg}$ and the number of anti-glaucoma medications used was $3.1 \pm 0.4$. Post-surgical mean IOP was $13 \pm 1 \mathrm{~mm}$ and number of medications was reduced to $0.8 \pm 1 .{ }^{42}$ This technique may hold promise in patients with chronically uncontrolled IOPs experiencing progressive glaucomatous damage. A 2014 study compared trabeculectomy with MMC, trabeculotomy and trabectome in patients with uveitic glaucoma. The surgical success rates were $82.86 \%, 62.5 \%$ and $75.0 \%$ for trabeculectomy, trabeculotomy, and trabectome, respectively ${ }^{43}$ The favorable comparison of trabeculectomy and trabectome further supports the value of trabectome in these cases. Although surgical intervention in patients with OAG secondary to PSS has been shown to be a viable management option, there are no consensus guidelines on when surgery should be pursued. Global risk assessment, progression analysis, individual patient and doctor risk-tolerance, and potential quality of life adjustments with and without surgical intervention should be considered prior to pursuing consultation for surgery.

\section{CONCLUSION}

PSS is a complex, poorly understood condition that may cause glaucomatous optic neuropathy, as demonstrated in our case. It is incumbent on the clinician to make an accurate diagnosis amongst multiple differential diagnoses, educate the patient to seek urgent care during cyclitic attacks to limit damage, assess IOP between attacks, monitor for the development and progression of secondary OAG, and appropriately refer for more aggressive interventions when medical management is unsuccessful. A commonly asked question is whether or not PSS is benign. Given the variability of this disease, its sequelae are patient-dependent and involve several factors, as outlined in this paper. In our case, PSS was not benign, which further underscored the need for vigilance in the longitudinal care of these patients to maintain functional vision and quality of life. •

\section{REFERENCES}

1. Posner A, Schlossman A. Syndrome of unilateral recurrent attacks of glaucoma with cyclitic symptoms. Arch Ophthalmol 1948;39(4):517-35.

2. Moorthy RS, Mermoud A, Baerveldt G, et al. Glaucoma associated with uveitis. Surv Ophthalmol 1997;41(5):361-94.

3. Shazly TA, Aljajeh M, Latina MA. Posner-Schlossman glaucomatocyclitic crisis. Semin Ophthalmol 2011;26(4-5):282-84.

4. Burnstein Y, Shelton K, Higginbotham EJ. Glaucomatocyclitic crisis in a child. Am J Ophthalmol 1998;126(1):136-7.

5. Meltzer MA, Friedman AH, Som PM, et al. Nonarteritic anterior ischemic optic neuropathy associated with acute glaucoma secondary to Posner-Schlossman Syndrome. Arch Ophthalmol 2017;121:127-8.

6. Jap A, Sivakumar M, Med M, et al. Is Posner Schlossman Syndrome benign? Ophthalmology 2001;108:913-8.

7. Chee SP, Jap A. Presumed Fuchs heterochromic iridocyclitis and Posner-Schlossman Syndrome: Comparison of cytomegaloviruspositive and negative eyes. Am J Ophthalmol 2008;146:883-9.

8. Megaw R, Agarwal PK. Posner-Schlossman syndrome. Surv Ophthalmol 2017;62(3):277-85.

9. Green RJ. Posner-Schlossman syndrome (glaucomatocyclitic crisis). Clin Exp Optom 2007;90(1):53-56.

10. Hung P, Chang J. Treatment of glaucomatocyclitic crises. Am J Ophthalmol 1974;77(2):169-72.

11. Bodh SA, Kumar V, Raina UK, et al. Inflammatory glaucoma. Oman J Ophthalmol 2011;4(1):3-9.

12. Kanda T, Shibata M, Taguchi M, et al. Prevalence and aetiology of ocular hypertension in acute and chronic uveitis. Br J Ophthalmol 2014;98:932-6.

13. Teoh SB, Thean L, Koay E. Cytomagalovirus in aetiology of PosnerSchlossman syndrome: evidence from quantative polymerase chain reaction. Eye 2005;19:1338-40.

14. Woo JH, Lim WK, Ho SL, et al. Characteristics of cytomegalovirus uveitis in immunocompetent patients. Ocul Immunol Inflamm 2015;23(5):378-83.
15. Rodier-Bonifas C, Cornut P, Billaud G, et al. Cytomegalovirus research using polymerase chain reaction in Posner-Schlossman syndrome. J Fr Ophthalmol 2011;34(1):24-9.

16. Chee SP, Bacsal K, Jap A, et al. Clinical features of cytomegalovirus anterior uveitis in immunocompetent patients. Am J Ophthalmol 2008;145:834-40.

17. Takusagawa HL, Liu Y, Wiggs JL. Infectious theories of PosnerSchlossman Syndrome. Int Ophthalmol Clin 2011;51(4):105-15.

18. Li J, Ang M, Cheung CMG, et al. Aqueous cytokine changes associated with Posner-Schlossman Syndrome with and without human cytomegalovirus. PLoS One 2012;7(9):e44453.

19. Hwang Y-S, Shen CR, Chang SHL, et al. The validity of clinical feature profiles for cytomegaloviral anterior segment infection. Graefe's Arch Clin Exp Ophthalmol 2011;249(1):103-10.

20. Vervoordeldonk MJBM, Tak PP. Cytokines in rheumatoid arthritis. Curr Rheumatol Rep 2002;4:208-17.

21. Haładyj E, Paradowska-Gorycka A, Felis-Giemza A, et al. Immunity and early atherosclerosis in the course of systemic lupus erythematosus, mixed connective tissue disease and antiphospholipid syndrome. Reumatologia 2016;4:187-95.

22. Shen SC, Ho WJ, Wu SC, et al. Peripheral vascular endothelial dysfunction in glaucomatocyclitic crisis: a preliminary study. Invest Ophthalmol Vis Sci 2010;51:272-6.

23. Zhao J, Zhu T, Chen W, et al. Human leukocyte antigens-B and -C loci associated with Posner-Schlossman Syndrome in a southern Chinese population. PLoS ONE 2015;10(7):e0132179.

24. Reijo A, Antti V, Jukka M. Endothelial cell loss in herpes zoster keratouveitis. Br J Ophthalmo 1983;67(11):751-4.

25. Cobo M, Foulks GN, Liesegang T, et al. Observations on the natural history of herpes zoster ophthalmicus. Curr Eye Res 1987;6(1):195-9.

26. Wensing B, Relvas LM, Caspers LE, et al. Comparison of rubella virus and herpes virus associated anterior uveitis: clinical manifestations and visual prognosis. Ophthalmology 2011;118(10):1905-10.

27. Mohamed Q, Zamir E. Update on Fuchs' uveitis syndrome. Curr Opin Ophthalmol 2005;16:356-63. 
28. Lee JR, Sung KR, Han S. Comparison of anterior segment parameters between the acute primary angle closure eye and the fellow eye. Invest Ophthalmol Vis Sci 2014;55(6):3646-50.

29. Muthusamy P. Apraclonidine in the management of glaucomatocyclitic crisis. Eye 1994;8 (Pt 3):367-8.

30. Hong C, Song K. Effect of apraclonidine hydrochloride on the attack of Posner-Schlossman syndrome. Korean J Ophthalmol 1993;7(1):28-33.

31. Harrington JR. Posner-Schlossman syndrome: a case report. J Am Optom Assoc 1999;70:715-23.

32. Controlled evaluation of loteprednol etabonate and prednisolone acetate in the treatment of acute anterior uveitis. Loteprednol Etabonate US Uveitis Study Group. Am J Ophthalmol 1999;127(5):537-44.

33. Pleyer U, Ursell PG, Rama P. Intraocular pressure effects of common topical steroids for post-cataract inflammation: are they all the same? Ophthalmol Ther 2013;2(2):55-72.

34. Park K, Hong C. Reversal of optic disc topography in patients with glaucomatocyclitic crisis after remission of attack. J Glaucoma 1998;7(4):225-9.

35. Darchuk V, Sampaolesi J, Lopez Mato O, et al. Optic nerve head behavior in Posner-Schlossman syndrome. Int Ophthalmol 2001;23:373-9.

36. Jampel H. Target pressure in glaucoma therapy. J Glaucoma 1997;6(2):133-8.
37. Wong VW, Km C, Dexter C, et al. Long-term results of oral valganciclovir for treatment of anterior segment inflammation secondary to cytomegalovirus infection. Clin Ophthalmol 2012;6:595-600.

38. Sobolewska B, Deuter C, Doycheva D, et al. Long-term oral therapy with valganciclovir in patients with Posner-Schlossman syndrome. Graefes Arch Clin Exp Ophthalmol 2014;252(1):117-24.

39. Chee SP, Jap A. Cytomegalovirus anterior uveitis: outcome of treatment. Br J Ophthalmol 2010;94(12):1648-52.

40. Su CC, Hu FR, Wang TH, et al. Clinical outcomes in cytomegalovirus-positive Posner-Schlossman Syndrome Patients treated with topical ganciclovir therapy. Am J Ophthalmol 2014;158:1024-31.

41. Boeckh M, Huang M, Ferrenberg J, et al. Optimization of quantitative detection of cytomegalovirus DNA in plasma by real-time PCR. J Clin Microbiol 2004;42(3):1142-8.

42. Pahlitzsch M, Torun N, Gonnermann J, et al. Trabeculectomy ab interno (trabectome): yet another possibility in the treatment of uncontrolled glaucomatocyclitic crisis under systemic valganciclovir therapy? Eye 2015;29:1335-9.

43. Shimizu A, Maruyama K, Yokoyama Y, et al. Characteristics of uveitic glaucoma and evaluation of its surgical treatment. Clin Ophthalmol 2014;26:2383-9. 